\title{
In-office endoscopic nasal polypectomy: prospective analysis of patient tolerability and efficacy
}

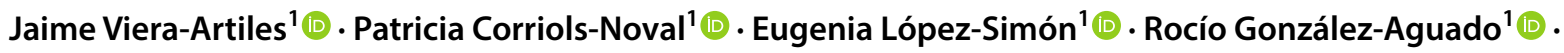 \\ David Lobo' ${ }^{1} \cdot$ Roberto Megía ${ }^{1}$ (i)
}

Received: 9 May 2020 / Accepted: 6 July 2020 / Published online: 14 July 2020

○) Springer-Verlag GmbH Germany, part of Springer Nature 2020

\begin{abstract}
Purpose In-office rhinologic procedures have become popularised in the last decade, especially in North America. Endoscopic nasal polypectomy under local anaesthesia offers instant relief in selected patients with obstructive chronic rhinosinusitis with nasal polyps. We aimed to analyse patient tolerability during the procedure while measuring its effectiveness. Methods A prospective study of patients who underwent in-office microdebrider-assisted polypectomy under local anaesthetic from September 2018 to November 2019 in a Spanish tertiary hospital was performed. The tolerability was measured by monitoring vital signs during the procedure and using a visual analogue scale posteriorly. The effectiveness was calculated through patient-reported outcomes (SNOT-22) and endoscopic evaluation 1 and 6 months follow-up.

Results Forty-four patients were included, with a mean age of 60.7 years. The mean visual analogue scale score was 2.76 out of 10 points. Vital signs were steady overall, with a statistically significant reduction $(p<0.001)$ in systolic pressure during the procedure. Presyncope and epistaxis were among the few mild complications. However, we registered one major complication that required intensive care admission. There was a 64\% reduction in the SNOT-22 score in the first month, with a maintained effect after 6 months. Patients with asthma and a higher polyp load were the subgroups that required more time to achieve significant improvement.

Conclusions In-office polypectomy is a very effective technique that alleviates obstructive symptoms in patients with nasal polyposis, and it is generally safe and well tolerated when performed by an expert. However, rhinologists must be aware of potentially severe complications.
\end{abstract}

Keywords Ambulatory surgical procedures $\cdot$ Nasal polyps/surgery $\cdot$ Otolaryngology/methods $\cdot$ Nasal obstruction/surgery . Patient satisfaction

\section{Introduction}

Nasal polyps are inflammatory outgrowths of sinonasal tissue that are estimated to affect $1-4 \%$ of the general US population, and they are frequently related to a subset of chronic rhinosinusitis, named chronic rhinosinusitis with nasal polyps (CRSwNPs) [1]. This entity is associated with significant morbidity and decreased quality of life, fundamentally caused by nasal obstruction and an alteration in sense of smell, which are the most severe and prevalent symptoms

Jaime Viera-Artiles

jaimevieraartiles@gmail.com

1 Otorhinolaryngology Department, Marqués Valdecilla University Hospital, Avenida Valdecilla s/n, Cantabria, Spain
[2]. The direct costs of functional endoscopic sinus surgery (FESS) in the USA range between $\$ 8,500$ and $\$ 11,000$. The highest costs are associated the expenses of repeated surgical procedures in patients with recurrent polyposis [3].

Whilst the initial management of CRSwNPs is based on topical and systemic therapies that depend on corticosteroids, surgery is considered after those have failed [1]. Traditionally, FESS has been performed in the operating room (OR) under general anaesthesia (GA) [4]. Nevertheless, some patients are reluctant to undergo surgery and reject it, while others can be rejected by the anaesthesiologist for high risk. Moreover, lengthy waiting list times in some healthcare systems can delay treatment and frustrate patients with severe symptoms [5].

Alternatively, removing nasal polyps under local anaesthesia (LA) in the office in selected chronic rhinosinusitis 
(CRS) patients with obstructive polyposis has become popularised in North America in recent decades [6, 7]. The objective of polypectomy is to re-establish normal nasal airflow and to improve the access of topical medication by removing polyps in a similar fashion as that done in the OR.

This technique seems to be cost effective [8] and has relatively long-standing beneficial results in the quality of life of patients [9]. However, the impact and expansion of this therapy in Europe has been very low in comparison to North America. Concerns about patient tolerability and safety could make clinicians hesitant about implementing this option in their clinical armamentarium.

In this study, we aimed to provide more in-depth evidence about patient experiences and safety while analysing the effectivity of the procedure.

\section{Materials and methods}

A descriptive prospective study of 44 consecutive patients who underwent office-based microdebrider-assisted polypectomy under LA from September 2018 to November 2019 in the Marqués de Valdecilla Hospital outpatient clinic was performed.

Adults with CRSwNPs and bilateral or unilateral (antrochoanal) obstructive nasal polyposis who had previously failed to respond to topical therapy with nasal corticosteroids and had tried at least one course of oral corticosteroids were considered for the in-office procedure. However, all patients had the option to undergo FESS (this option was recommended to those with more intense sinus involvement in the CT scans). The following inclusion criteria were also included: (1) patients who preferred use of LA for polyp removal over FESS under GA after an explanation of the different benefits and risks of both procedures; (2) patients dismissed by the anaesthesiologist to undergo GA due to high risk; and (3) patients awaiting elective FESS with severe CRSwNPs and complete blockage of both nostrils who were anxious about the nasal obstruction. On the other hand, exclusion criteria were the following: (1) patients with anxiety disorders or those who did not tolerate the endoscopic exam in the clinic; (2) patients with major comorbidities (mainly cardiological or respiratory pathologies); or (3) patients allergic to LA.

Patient demographics and their medical histories were collected. The severity of the pathology was determined endoscopically using the Lund-Kennedy (LK) scale and clinically using the validated Spanish version of the SNOT22 questionnaire [10] in all participants before the intervention. The Lund-MacKay (LM) scale was only used in patients with a previous $\mathrm{CT}$ scan performed within the last 6 months.
The polypectomies were performed by three different trained rhinologists using the same technique. The intraoperative details and complications were collected in every case. Patient tolerance was measured using vital sign monitoring and the visual analogue scale (VAS), querying for pain after the intervention.

In the first follow-up appointment 1 month after the procedure, the patients were inquired for complications (epistaxis, dizziness, nausea, etc.) and asked directly if they would have the procedure done again if needed. Clinical outcomes were estimated through endoscopic evaluation and through the SNOT-22 questionnaires in the first and second follow-up appointment, 1 and 6 months, respectively.

Using StataCorp. 2013. Stata Statistical Software: Release 13. (College Station, TX: StataCorp LLC.) We performed a descriptive analysis of all different variables of the sample. We used Student's $t$-test to analyse the intrasubject variation of vital signs during the procedure and the variation in the endoscopic evaluation using the LK scale. Finally, we built a predictive model of the clinically reported outcomes of the whole sample using logistic regression and other predictive models of the different subgroups.

\section{Room set-up, patient preparation, and surgical technique}

The procedures were performed in a regular otorhinolaryngology office at our outpatient clinic with the assistance of a trained nurse. The study was conducted prior to coronavirus outbreak, so no special personal protective equipment (PPE) was used. All patients were in a seated position on a recliner chair. Intravenous accesses were placed in the forearm as a safety measure, and vital signs were monitored before the procedure started. The electrically powered microdebrider console and the endoscopic monitor were usually located at the right-hand side of the surgeon, and the vital sign monitor was located at the opposite side. Continuous vacuum suction was always available.

Preparation of the nose was done using surgical patties soaked in a solution of oxymetazoline and lidocaine for at least $5 \mathrm{~min}$. Consecutively, a mixture of $2 \%$ articaine and 1:200,000 epinephrine was injected submucosally in the lateral nasal wall and in the "agger mount," above the armpit of the middle turbinate. Also, specific regions, such as septal spurs, were infiltrated using a local anaesthetic syringe (breed loading, metallic, cartridge type syringe). No sedation was used. Patients' blood pressure, oxygen saturation, and heart rate were monitored throughout the procedure.

Under endoscopic vision using a HOPKINS ${ }^{\circledR} 4.0 \mathrm{~mm}$ and 0-degree rigid endoscope (KARL STORZ, Tuttlingen, Germany), the electric Straightshot ${ }^{\mathrm{TM}}$ M5 microdebrider (Medtronic. Minnesota, USA) with a suction irrigation system was inserted into the nasal cavity, and the polyps 
were removed while trying to preserve the normal structures and healthy mucosa. In cases of extensive polyposis, it was often necessary to sequentially decongest and anaesthetise the nasal cavity as the dissection proceeded. Biopsy samples were sent to the pathology laboratory for diagnosis. No nasal packing was used afterwards.

Previously and after the procedure, the same medical therapy based on corticosteroid-impregnated nasal irrigation $(2 \mathrm{~mL}$ of $0.5 \mathrm{mg} / \mathrm{mL}$ budesonide ampoules in $250 \mathrm{~mL}$ of saline) once daily or twice daily was prescribed to all patients, depending on the severity of the mucosal inflammation.

\section{Results}

A total of 44 patients from September 2018 to November 2019 were included in this study, with $73 \%$ males $(n=32)$ and a mean age of 60.7 years (range of 25-83 years). The follow-up time points for this study were 1 and 6 months. However, we continued passing questionnaires after the second follow-up to the first participants, which resulted in a mean global follow-up time of 9.6 months.

The most common indication was obstructive CRSwNPs after failure of the medical therapy (93.3\%), and the rest of the patients $(7.7 \%)$ had a unilateral or "antrochoanal" polyp. Of the patients, $28.8 \%$ have previously had an average of 1.75 FESS or polypectomies under GA, and $15.5 \%$ of the patients with massive polyposis were included to relieve nasal obstruction prior to a standard FESS.

The most common comorbidity was asthma (46.5\%), either alone $(32.6 \%)$ or in combination with aspirin intolerance (13.9\%) as part of Samter's triad. The rest of the patients' previous conditions are summarised in Table 1.

Three out of 44 patients abandoned the study after the procedure. One patient abandoned after a partial polypectomy due to repeated presyncopes. The second patient had a unilateral polyp that ended up being an inverted papilloma; therefore, she was removed from the study and scheduled for

Table 1 Summarize of the patients' characteristics

\begin{tabular}{ll}
\hline Gender & $71 \%$ males $(n=32)$ \\
& $29 \%$ females $(n=13)$ \\
Age (years) & $25-83(60.7$ mean $)$ \\
HBP & $25.6 \%$ \\
Asthma & $46.5 \%$ \\
Samters Triad & $13.9 \%$ \\
Previous surgery & $28.8 \%$ \\
OAs & $6.6 \%$ \\
LK scale preop & $6.3 / 12$ mean \\
LM score preop & $11 / 24$ mean \\
Previous CT scan & $53.3 \%$ \\
\hline
\end{tabular}

expanded surgery. However, both patients were included in the tolerability analysis. The third patient had a severe syncope during the polypectomy and the procedure was aborted.

\section{Patient tolerance}

Patient tolerance was measured in the 43 patients who had a polypectomy. Objectively, there were minor variations in heart rate and oxygen saturation measurements pre-surgery, during surgery, and post-surgery, with some of them being statistically significant (Table 2). Nevertheless, the intraoperative and postoperative intra-subject decreases in systolic blood pressure were the most important statistically significant variations (Fig. 1).

Subjectively, using the VAS after surgery for the question, "How painful was the procedure?" resulting in a mean value of 2.76 and a median of 3 out of 10 points (range of $0-7)$, where 0 means total absence of pain, and 10 means the most intense pain they have ever felt. The most common description for the unpleasant sensation was "like a sting" or "like a bite," expressing a very brief period of intense but bearable pain.

Anecdotally, patients with lower tolerance (VAS $>4$ ) had higher average LK (8.13 vs 5.91 in VAS $<4)$ and LM scores (12 vs 10.71 in VAS $<4)$. Also, patients with asthma had almost one VAS point of difference with those without

Table 2 Blood pressure, heart rate and oxygen saturation mean comparison results using Student's $t$ test, pre-surgically, intra-surgically and post-surgically

\begin{tabular}{lllr}
\hline & Mean difference & CI 95\% & $p$ value \\
\hline SBP & & & \\
Pre-Int & 9.24 & $1.36-17.11$ & 0.02 \\
Int-Pos & 1.81 & $-4.9-8.52$ & 0.59 \\
Pre-Pos & 11.23 & $5.29-17.18$ & $<0.001$ \\
DBP & & & \\
Pre-Int & 3.67 & $-0.18-7.51$ & 0.06 \\
Int-Pos & 0.57 & $-5.42-6.56$ & 0.85 \\
Pre-Pos & 4.53 & $-2.18-11.25$ & 0.18 \\
HR* & & & \\
Int-Pre & 2.64 & $0.03-5.25$ & 0.05 \\
Pos-Int & 1.1 & $2.61-4.8$ & 0.55 \\
Pos-Pre & 3.37 & $0.32-6.42$ & 0.03 \\
O 2 Sat & & & \\
Pre-Int & 0.44 & $0.1-0.79$ & 0.01 \\
Int-Pos & 3.26 & $-2.85-9.36$ & 0.29 \\
Pre-Pos & 3.7 & $-2.41-9.81$ & 0.23 \\
\hline
\end{tabular}

SBP systolic blood pressure, DBP diastolic blood pressure, HR heart rate, $\mathrm{O}_{2}$ Sat oxygen saturation, Pre pre-surgical, Int intra-surgical, Pos post-surgical, CI confidence interval

*The order of the comparisons was switched to make the values positive, as the HR slightly increased during the procedure 


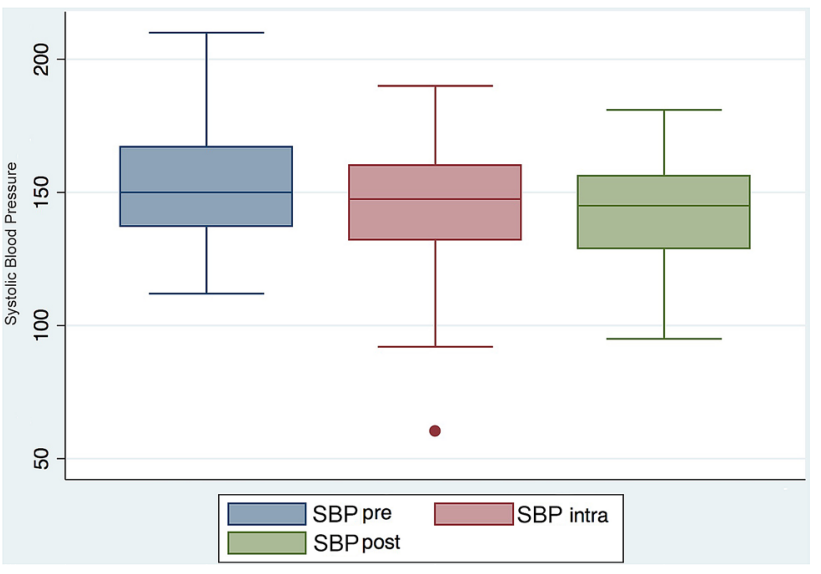

Fig. 1 Systolic blood pressure's box plot pre-surgery, during surgery, and post-surgery

asthma (3.2 vs 2.37 , respectively). Moreover, patients in which a surgeon with more experience in polypectomies intervened $(n=23)$ had a lower mean VAS score than those in which a surgeon with less experience intervened $(n=8)$ ( 2.57 vs 3.5 , respectively). On the other hand, there were almost no differences in the VAS score regarding sex, age, previous interventions, and indications for the procedure. These results have no statistical significance due to the small number of participants in each subgroup and the lack of a control group.

Only three patients (6.8\%) did not tolerate the treatment. The first two have been already explained (repeated presyncopes and severe syncope) and the third one had massive polyposis and was waiting for a FESS under GA, he only had a partial polypectomy due to discomfort (VAS $=7 / 10$ ).

Overall, the patients had a good experience. In the second follow-up appointment, $92.5 \%$ expressed that they would have the procedure repeated if it was needed.

\section{Complications}

The most common intraoperative complication was the presyncope, which occurred in four patients $(9.1 \%)$, three males and one female, with a mean age of 60.7 years. However, three of them were able to finalise the procedure following the Trendelenburg manoeuvre and resting for several minutes.

There were only two patients with intraoperative bleeding (one in the olfactory groove area and the other in a middle turbinate by the feeding branch from the sphenopalatine artery), which required immediate cauterisation in the office. In the rest of the patients, mucosal bleeding was managed using oxymetazoline-impregnated cottonoid patties and vacuum suction.
We registered a major intraoperative complication in a polymedicated 77-year-old male with a previous history of hypertension and asthma. He experienced severe bradycardia and bradypnea, quickly followed by decreased consciousness during polyp removal, requiring urgent oral endotracheal intubation and advanced cardiac life support. Then, he was admitted to the intensive care unit, and a brain CT scan, electroencephalogram, and electrocardiogram were performed to rule out cerebral ischemia, a vascular event, or heart disorders. No critical findings were found; the patient recovered several hours later and was discharged within $48 \mathrm{~h}$ without any sequels.

Postoperatively, the most common complaint was epistaxis, which was only registered in two patients (4.5\%). Both epistaxis were easily solved using anterior gauze packing in the emergency room by the ENT resident on call.

In the first follow-up interview, there were no reported complaints about pain, discomfort, nausea, or dizziness in the days following the procedure. Patients resumed their normal activities (either at work or at home) in a mean time of 1.5 days afterwards.

\section{Effectivity}

The effectivity of the procedure was measured in 41 patients. The mean pre-surgery SNOT-22 score was 40.8 [standard deviation $(\mathrm{SD})=21.4]$, and the mean score in the first follow-up appointment 1 month after the procedure decreased $64 \%$, down to $14.7(\mathrm{SD}=10.5)$. The mean score at 6 months' follow-up was 11.2.

In the regression analysis, the independent variable is the SNOT-22 pre-surgery and the dependent variables are the SNOT-22 score at 1-month and 6-month post-surgery, as shown in Fig. 2. The predictive models in both time points were statistically significant. The pre-surgery SNOT-22 regression coefficient is 0.19 (95\% CI 0.04-0.34), which means that there is a positive linear association with the first follow-up SNOT-22 scores $(p=0.01)$ [Fig. 2a]. Similarly, the second follow-up SNOT-22 scores have a positive and significant $(p<0.001)$ linear association in with the presurgery SNOT-22 which regression coefficient is 0.25 (95\% CI 0.12-0.37) [Fig. 2b].

The predictive model using the linear regression was also statistically significant for patients with asthma, a high LK score $(>6)$, a high LM score $(>16)$, or patients with previous surgical interventions (FESS or polypectomy under GA), but only at the second follow-up appointment (Table 3).

Endoscopically, there was a mean difference between presurgical LK score and post-surgical LK of 4.66 points (95\% CI 3.65-5.69) in the first follow-up, which was statistically significant $(p<0.001)$ (Fig. 3).

Most patients had a complete removal of the nasal polyps (including four patients with complete anterior and partial 

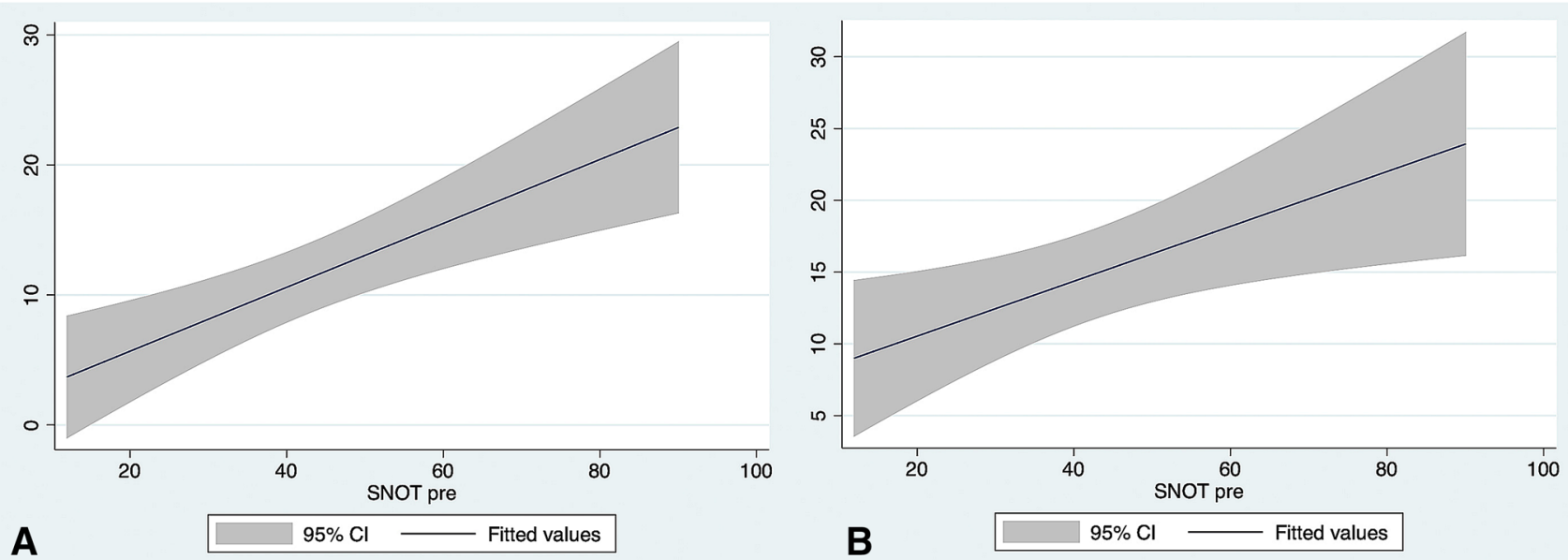

Fig. 2 SNOT-22 score predictive models using the linear regression in the first follow-up at 1 month (a) and the second follow-up at 6 months (b). The ordinate axis indicates the SNOT-22 score prediction

Table 3 Subgroup linear regression models in the first and second follow-up ( 1 and 6 months, respectively)

\begin{tabular}{|c|c|c|c|c|c|c|c|c|c|c|c|c|}
\hline & \multicolumn{3}{|c|}{ Asmathic patients } & \multicolumn{3}{|c|}{ Lund-Kennedy $>6$} & \multicolumn{3}{|c|}{ Lund-Mackey > 16} & \multicolumn{3}{|c|}{ Previous surgeries } \\
\hline & $\mathrm{RC}$ & $95 \% \mathrm{CI}$ & PV & $\mathrm{RC}$ & $95 \% \mathrm{CI}$ & PV & $\mathrm{RC}$ & $95 \% \mathrm{CI}$ & PV & $\mathrm{RC}$ & $95 \% \mathrm{CI}$ & PV \\
\hline First FU & 0.22 & $-0.05-0.49$ & 0.11 & 0.19 & $-0.01-0.38$ & 0.06 & 0.26 & $0.01-0.5$ & 0.04 & 0.25 & $-0,05-0.55$ & 0.09 \\
\hline Second FU & 0.32 & $0.09-0.55$ & 0.01 & 0.29 & $0.12-0.45$ & 0.001 & 0.29 & $0.09-0.5$ & 0.008 & 0.27 & $0.09-0.46$ & 0.01 \\
\hline
\end{tabular}

$\mathrm{RC}$ regression coefficient, $\mathrm{PV} p$ value, FU follow-up

Fig. 3 a Right nasal fossa completely obstructed by polyps. b The same patient 6 months after in office polypectomy
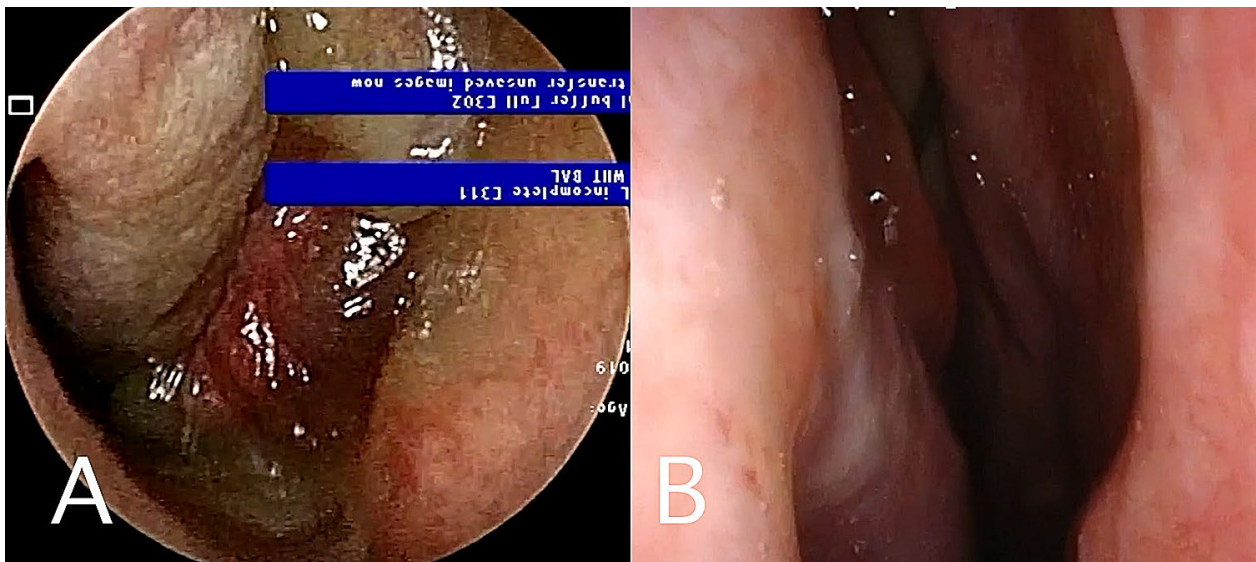

posterior ethmoidectomy) and only six patients had a partial polypectomy. The mean SNOT-22 reduction was greater in this last group in the first follow-up (42.8 points reduction versus 24.5 points reduction in the first group) but the SNOT-22 scores improved in the last follow-up only in the complete-removal group. However, due to the small number of the subgroup analysis, the results were not statistically significant.

Of the patients, $16.6 \%$ had polyp regrowth. Nevertheless, there was a $63.6 \%$ reduction in their SNOT-22 score at the second follow-up. Only one patient experienced almost no improvement and was subsequently scheduled for FESS.

\section{Discussion}

Endoscopic intranasal polypectomy is a complex procedure that requires a great deal of experience in the operating room to be performed safely in the office. The main goal of this treatment is to clear the nasal passages from obstructive 
polyps and restore nasal breathing. However, when selecting a patient, other factors besides the polyps' degree should be considered. A proper selection can make this procedure comfortable and successful for both the patient and the surgeon, avoiding potential severe complications.

Invasive medical procedures with a VAS score lower than 4 are considered well tolerated [11]. The mean VAS score in this sample was 2.76, showing that in-office endoscopic polypectomy by experts is perceived as tolerable by most patients. Furthermore, consistent vital signs throughout procedures reinforce this statement. Although this is a subjective test and every patient might have a different pain threshold, the low mean score obtained in this study seems to be in concordance with the overall experience the patients had. Moreover, the vast majority expressed that in case of necessity they would have the polypectomy done again in the future, and they were thankful for the treatment and symptoms relief.

Contrary to our predictions, instead of an increase in systolic pressure, there was a decrease during the procedure. This might be explained by the relaxation of previously anxious patients after realising that polypectomy was less painful than they expected.

Conversely, patients with asthma, a higher polyps load, and mucosal inflammation (higher LK and LM scores) seem to have a lower tolerance to the procedure, but this was not confirmed statistically. A possible explanation is the combination of a less effective anaesthetic infiltration of the inflamed mucosa and increased mucosal bleeding. Therefore, primary patients with massive polyposis might not be good candidates for in-office polypectomy.

Even though the procedure is well tolerated, it is not free from complications. Whilst most are mild to moderate and can be solved instantly (e.g., bleeding or presyncope), complications such as severe syncope might occur. Hence, performing this technique in a tertiary hospital with an ICU available is highly recommended.

The question arises if this procedure is really safe when one out of forty-four patients (2.27\%) of severe complications happened in this study. The author experience goes beyond this study and there are also other series published in the literature related to in-office polypectomy where none of them have registered this complication so far. Nevertheless, there are several case reports of severe hypotension during nasal or oral manipulations that could be explained by the trigeminal cardiac reflex (TCR), triggered by the stimulation of any branch of the fifth cranial nerve along its course [12]. It is a clinical phenomenon characterised by hemodynamic alterations such as hypotension, apnoea, bradycardia, and gastric hypermobility [13]. It represents one of the most powerful autonomous reflexes, and it has been infrequently noted by otolaryngologists. The strength and duration of the stimulus in combination with other risk factors (e.g., medication) have been described as triggers for the TCR. It may be elicited without prior hemodynamic changes; therefore, it is important to be aware and to anticipate its onset [14].

Patients with anticoagulant or antiaggregant had the medication suspended days before the procedure and there was no increased epistaxis' ratio in those patients. Therefore, the use of blood thinners is not a risk factor for increased intraoperative bleeding if those are suspended previously.

From the authors' perspective, tolerance of this procedure is based on a combination of the painless removal of nasal polyps, good bleeding control, and a relaxed patient. This can be achieved by considering the following three main points:

\section{Patient selection}

The first interview and the physical exams are extremely useful to determine if a patient could bear an in-office polypectomy. The intolerance due to anxiety related to the nasal endoscopic exam or a previous history of presyncope during medically invasive acts could make those patients unsuitable for polypectomy under LA. Anti-anxiety medication before the procedure could be useful, however, we did not use it in any patient.

\section{Local anaesthetic}

Polyps are devoid of sensitive fibres. Therefore, anaesthesia should target the lateral walls and middle turbinate mucosa. Septal deviations or spurs might interfere with polyp removal; hence, infiltrating those areas is recommended to achieve a painless procedure. Different anaesthesia protocols can be found in the literature. On one hand, Krouse et al. sprayed the nasal cavity with $3 \%$ ephedrine plus $2 \%$ tetracaine, and then $1 \%$ xylocaine with 1:100,000 epinephrine was injected into the base of the polyps [6]. On the other hand, Eng Cern et al. only used 4\% topical xylocaine and $0.05 \%$ oxymetazoline spray [15]. However, we believe that the surgical technique is more important in improving patient's comfort than the anaesthesia itself. Care must be taken when removing polyps in the posterior part of the nasal cavity, such as the sphenoethmoidal recess, because it is a more difficult part to reach with the anaesthetic. A spinal needle can be used to infiltrate this area.

\section{Surgical technique}

Removing only nasal polyps while respecting healthy mucosa reduces bleeding and pain. To minimise bleeding and to improve visualisation, mucosal decongestion with vasoconstrictors is highly recommended. The vacuum suction of the electric microdebrider will help prevent the 
patient from swallowing an excessive amount of blood and it will control nausea. Olfactory cleft polyps seem to bleed more, probably because they are mostly respiratory epithelial adenomatoid hamartomas (REAHs) instead of eosinophilic polyps and result in a more painful resection; thus, we do not recommend removing them thoroughly. The dissection of the anterior and posterior ethmoids can be performed in patients with favourable anatomy and good tolerance to the procedure. We also have to bear in mind that this technique has its own learning curve, which can be seen in the lower VAS scores obtained by the surgeon with more experience.

Using the predictive model, it was shown that the effectiveness of the procedure was statistically significant improving patient-reported outcomes at the first follow-up (1 month), with mildly decreased patient-reported outcomes at the second follow-up (6 months), probably because the patients got used to their new breathing status and some of them had polyp regrowth. These results are similar to other published studies [16] and comparable with polypectomy under GA [17]. In addition, the statistically significant reduction in the postoperatively LK score reinforces this subjective improvement, in this case, through the surgeon's endoscopic evaluation.

In opposition, patients with asthma, higher LK or LM scores, or previous surgical nasal interventions had only statistically significant outcomes in the second follow-up, which could be explained by the longer period required for the budesonide irrigations to reduce the more severe global inflammation in those patients.

Contrary to our predictions, we did not find significant differences in symptoms relieve in patients who have previously had FESS versus primary cases. This could be explained by the better access for topical medication inside the paranasal sinuses mucosa once the obstructive polyps were removed, even though the sinuses themselves were not addressed.

Patients who had partial polypectomy had bigger SNOT22 score reduction than those who had complete polyps' resection in the first follow-up. This could be explained because those first patients usually had greater polyp load and were more symptomatic than the second ones, obtaining greater symptoms relieve in the short term. However, the SNOT-22 scores only kept improving in those who had a complete resection. This effect was even better in those with additional partial ethmoidectomy, probably due to a better access of the medication into the sinuses.

\section{The ideal patient}

It seems that in-office polypectomy is an effective treatment for obstructive polyposis in selected patients. From our results, we can say that the ideal patients are those with moderate polyp load and moderate inflammation with nasal obstruction as main symptom. Conversely, patients with more severe pathology and anosmia or recurrent infections as predominant symptoms will usually require surgery under GA with complete sinus clearance. However, some of these patients might reject surgery and we have demonstrated than they can tolerate this procedure and obtain great benefit from in-office polypectomy, even though it is not the ideal treatment for them. Clearing the nasal passages also allows better delivery of intranasal corticosteroids which will help improving other sinonasal symptoms.

Even though our inclusion criteria are similar to other publications, future randomized studies should focus on defining the real role of this procedure in the treatment of CRSwNPs. It should be clarified which is the most costeffective treatment and which type of CRSwNP patient will obtain the longest term benefit after gathering long-term results and comparing polypectomy with other therapeutic alternatives. However, with the current data we can say that in-office polypectomy in selected patients is a good alternative to FESS to be considered in healthcare systems with increased waiting lists. Additionally, it is also a great option for patients with polyps' regrowth after previous FESS and in unoperated patients with obstructive polyposis and moderate sinus involvement.

Another advantage is that CT scans are not mandatory if the patient decides not to have surgery under GA. This will speed up the treatment in saturated healthcare systems where the waiting list for a CT scan can add up to the surgical waiting list, resulting in more frustrated patients.

The ongoing COVID-19 pandemic is changing the way rhinologic procedures should be done. The role of the nasal and paranasal sinus cavities is increasingly recognized for COVID-19 symptomatology and transmission. Therefore, the importance of taking extra precautions to safely perform rhinological procedures and examinations. When performing a potential aerosol and respiratory droplets generating procedure like in-office polypectomy, the currently available guideline recommendation for personal protective equipment should be followed [18].

To our knowledge, this is the first study to report patient experience and nasal outcomes under endonasal in-office polypectomy in Spain and the largest sample using an electric microdebrider globally to date. Eng Cern et al. highlight the advantages of the vacuum-powered microdebrider versus the electric in terms of comfort and time-saving [15]. However, we have shown that the electrically powered microdebrider is also well tolerated.

The limitations of this study included the lack of a direct control group and the size of the sample, which only allowed us to perform a descriptive analysis of patient subjective tolerability. The relatively short follow-up period for the effectivity analysis could also be a weakness when trying to provide evidence about long-term results. Besides, there 
might also be a selection bias as anxious patients might have chosen FESS more often than in-office polypectomy, when the two options were offered. Moreover, the surgeons might have had a tendency to suggest FESS in severe cases and polypectomy in milder cases. However, in the final sample there were cases from all the severity spectrum of this disease.

\section{Conclusion}

In-office polypectomy is a safe and well-tolerated procedure when performed by an expert. It is also very effective, alleviating frustrating symptoms in CRSwNP patients. However, it should be performed by rhinologists in a tertiary hospital setting since severe complications might occur. This procedure could fill a therapeutic gap between the standard surgical and medical treatments, offering clinicians and patients a good alternative to treat obstructive nasal polyposis even in severe cases, especially in saturated healthcare systems. Nevertheless, further randomized studies are needed to narrow down the most cost-effective indications.

\section{Compliance with ethical standard}

Conflict of interest All authors declare there is no conflict of interest in this publication

\section{References}

1. Stevens WW, Schleimer RP, Kern RC (2016) Chronic Rhinosinusitis with nasal polyps. J Allergy Clin Immunol Pract 4(4):565572. https://doi.org/10.1016/j.jaip.2016.04.012

2. Dietz De Loos DAE, Hopkins C, Fokkens WJ (2013) Symptoms in chronic rhinosinusitis with and without nasal polyps. Laryngoscope 123(1):57-63. https://doi.org/10.1002/lary.23671

3. Fokkens WJ, Lund VJ, Hopkins C (2020) European Position Paper on Rhinosinusitis and Nasal Polyps. Rhinol J. 58:1-464 (Supplement 29)

4. Tajudeen BA, Kennedy DW (2017) Thirty years of endoscopic sinus surgery: what have we learned? World J Otorhinolaryngol Head Neck Surg 3(2):115-121. https://doi.org/10.1016/j.wjorl .2016.12.001

5. Tsang GFZ, McKnight CL, Kim LM et al (2016) Exploring the psychological morbidity of waiting for sinus surgery using a mixed methods approach. J Otolaryngol Head Neck Surg 45(1):17. https://doi.org/10.1186/s40463-016-0149-z

6. Krouse JCD (1996) Powered nasal polypectomy in the office setting. Ear Nose Throat J 75(9):608-610

7. Scott JR, Sowerby LJ, Rotenberg BW (2017) Office-based rhinologic surgery: a modern experience with operative techniques under local anesthetic. Am J Rhinol Allergy 31(2):135-138. https ://doi.org/10.2500/ajra.2017.31.4414

8. Rudmik L, Smith KA, Kilty S (2016) Endoscopic polypectomy in the clinic: a pilot cost-effectiveness analysis. Clin Otolaryngol 41(2):110-117. https://doi.org/10.1111/coa.12473

9. Coates ML, Mayer A, Carrie S (2020) Developing an innovative office-based UK rhinology service-experience and outcomes in 22 patients undergoing office-based local anaesthetic nasal polypectomy. Clin Otolaryngol 45(2):268-273. https://doi. org/10.1111/coa.13487

10. de losSantos G, Reyes P, del Castillo R et al (2015) Cross-cultural adaptation and validation of the sino-nasal outcome test (SNOT22) for Spanish-speaking patients. Eur Arch Oto-Rhino-Laryngol. 272(11):3335-3340

11. Jensen MP, Chen C, Brugger AM (2003) Interpretation of visual analog scale ratings and change scores: a reanalysis of two clinical trials of postoperative pain. J Pain 4(7):407-414. https://doi. org/10.1016/S1526-5900(03)00716-8

12. Arasho B, Sandu N, Spiriev T et al (2009) Management of the trigeminocardiac reflex: facts and own experience. Neurol India 57(4):375-380. https://doi.org/10.4103/0028-3886.55577

13. Radvansky BM, Husain Q, Cherla DV et al (2013) In-office vasovagal response after rhinologic manipulation. Int Forum Allergy Rhinol 3(6):510-514. https://doi.org/10.1002/alr.21121

14. Meuwly C, Golanov E, Chowdhury T et al (2015) Trigeminal cardiac reflex: new thinking model about the definition based on a literature review. Medicine (United States) 94(5):e484. https:// doi.org/10.1097/MD.0000000000000484

15. Gan EC, Habib ARR, Hathorn I et al (2013) The efficacy and safety of an office-based polypectomy with a vacuum-powered microdebrider. Int Forum Allergy Rhinol 3(11):890-895. https:// doi.org/10.1002/alr.21198

16. Coates ML, Carrie S (2019) A local safety standard for invasive procedures for out-patient endonasal procedures performed under local anaesthetic: a template from Newcastle upon Tyne hospitals. J Laryngol Otol 133(5):441-444. https://doi.org/10.1017/S0022 215119000781

17. Kilty SJ, Lasso A, Mfuna-Endam L et al (2018) Case-control study of endoscopic polypectomy in clinic (EPIC) versus endoscopic sinus surgery for chronic rhinosinusitis with polyps. Rhinology 56(2):155-157. https://doi.org/10.4193/Rhin17.115

18. Tysome JR, Bhutta M (2020) COVID-19: protecting our ENT workforce. Clin Otolaryngol. https://doi.org/10.1111/coa.13542

Publisher's Note Springer Nature remains neutral with regard to jurisdictional claims in published maps and institutional affiliations. 